\title{
VEGF-PLC $\gamma 1$ pathway controls cardiac contractility in the embryonic heart
}

\author{
Wolfgang Rottbauer, ${ }^{1,2,4,6}$ Steffen Just, ${ }^{1,4}$ Georgia Wessels, ${ }^{1}$ Nicole Trano, ${ }^{1}$ Patrick Most, ${ }^{1}$ \\ Hugo A. Katus, ${ }^{1}$ and Mark C. Fishman ${ }^{2,3,5}$ \\ ${ }^{1}$ Department of Medicine III, University of Heidelberg, D-69120 Heidelberg, Germany; ${ }^{2}$ Cardiovascular Research Center, \\ Massachusetts General Hospital and Department of Medicine, Harvard Medical School, Boston, Massachusetts 02114, USA; \\ ${ }^{3}$ Novartis Institutes for BioMedical Research, Cambridge, Massachusetts 02139, USA
}

\begin{abstract}
The strength of the heart beat can accommodate in seconds to changes in blood pressure or flow. The mechanism for such homeostatic adaptation is unknown. We sought the cause of poor contractility in the heart of the embryonic zebrafish with the mutation dead beat. We find through cloning that this is due to a mutation in the phospholipase $\mathrm{C} \gamma 1$ (plc $\gamma 1)$ gene. In mutant embryos, contractile function can be restored by PLC $\gamma 1$ expression directed selectively to cardiac myocytes. In other situations, PLC $\gamma 1$ is known to transduce the signal from vascular endothelial growth factor (VEGF), and we show here that abrogation of VEGF also interferes with cardiac contractility. Somewhat unexpectedly, FLT-1 is the responsible VEGF receptor. We show that the same system functions in the rat. Blockage of VEGF-PLC $\gamma 1$ signaling decreases calcium transients in rat ventricular cardiomyocytes, whereas VEGF imposes a positive inotropic effect on cardiomyocytes by increasing calcium transients. Thus, the muscle of the heart uses the VEGF-PLC $\gamma 1$ cascade to control the strength of the heart beat. We speculate that this paracrine system may contribute to normal and pathological regulation of cardiac contractility.
\end{abstract}

[Keywords: VEGF; PLC $\gamma 1$; contractility; heart; zebrafish]

Supplemental material is available at http://www.genesdev.org.

Received March 30, 2005; revised version accepted May 23, 2005.

In vertebrates, the heart and vasculature have great ability to match function to hemodynamic needs. Some of this is due to homeostatic mechanisms extrinsic to the heart. For example, sympathetic nerves and circulating catecholamines enhance contractility in the face of fluid loss. There is, additionally, a powerful system intrinsic to the heart that matches precisely the input to output of the heart. This so-called Frank-Starling relationship, first described in 1918, is manifest even in an isolated heart (Starling 1918). The molecular basis of this intrinsic system is not known. It has been speculated to reflect better alignment of the myofibrillar arrays (Sonnenblick et al. 1973) or regulation of intracellular calcium $\left(\mathrm{Ca}^{2+}\right)$ (Gordon and Pollack 1980; Van Henningen et al. 1982; Gillebert et al. 1989|. Even the embryonic heart manifests a Frank-Starling response well before innervation (Benson et al. 1989).

Phospholipase C (PLC) hydrolyzes phosphatidylinositol 4,5-bisphosphate $\left(\mathrm{PIP}_{2}\right)$, producing two second mes-

\footnotetext{
${ }^{4}$ These authors contributed equally to this work. Corresponding authors.

${ }^{5}$ E-MAIL mark.fishman@pharma.novartis.com; FAX (617) 871-3402. ${ }^{6}$ E-MAIL wolfgang.rottbauer@med.uni-heidelberg.de; FAX 0049-6221564886.

Article and publication are at http://www.genesdev.org/cgi/doi/10.1101/ gad.1319405.
}

sengers, inositol 1,4,5-trisphosphate $\left(\mathrm{IP}_{3}\right)$ and diacylglycerol (DAG). $\mathrm{IP}_{3}$ induces a transient increase in intracellular free $\mathrm{Ca}^{2+}$, while DAG directly activates protein kinase C (PKC) (Lamers et al. 1993). To date, 11 mammalian PLC-isozymes have been characterized. Targeted disruption of the $p l c \gamma 1$ gene in mice leads to severe defects in blood-vessel formation (Liao et al. 2002). y10 zebrafish mutants, which have recently shown to be at least partially deficient in PLC $\gamma 1$ have defects in formation of the artery, but not the vein (Lawson et al. 2003).

Vascular endothelial growth factor (VEGF) acts via its receptors FLT-1 and FLK- 1 to activate PLC $\gamma 1$. In the embryo, this activity is essential to establish and maintain the earliest vessels, a process termed vasculogenesis, as well as to direct later sprouting, termed angiogenesis. Disruption of VEGF or its receptors is early embryonic lethal because of this critical role (Neufeld et al. 1999). VEGF is produced by the myocardium, where one role clearly is to enhance vascularization. Indeed, its elimination in the heart prevents vessel formation (Ferrara 2001).

Here, we show that the recessive lethal zebrafish mutation, dead beat (ded) (Stainier et al. 1996), loses contractility of its ventricle and lacks a lumenized vasculature. By positional cloning, we show that the ded mutation is in zplcy1. Cardiac-specific expression of wild-type 
zplcy1 in ded mutant cardiomyocytes restores contractility, even in the absence of blood circulation. We demonstrate that VEGF via its receptor FLT-1 acts upstream of PLC $\gamma 1$ to control cardiac ventricular contractility. Using cultured rat neonatal ventricular cardiomyocytes, we show VEGF-FLT-1-PLC $\gamma 1$ signals to control contractility in a reversible and rapid manner by modifying cardiomyocyte calcium cycling. These observations indicate that VEGF, its receptor FLT-1, and $z$ PLC $\gamma 1$ are key components of a pathway that controls the onset and maintenance of cardiac ventricular contractility, independently of its impact on vessel formation.

\section{Results}

Zebrafish dead beat (ded) renders the cardiac ventricle noncontractile, and affects key steps in vasculogenesis

We isolated the zebrafish mutation dead beat ( ded $^{\text {m582) }}$ in a large-scale ENU-mutagenesis screen for recessive lethal mutations that perturb cardiac function (Stainier et al. 1996). ded mutant embryos display progressive, ventricle-specific reduction of cardiac contractility. Aside from pericardial edema, ded embryos are not noticeably affected by the lack of normal blood flow during the first week of development (Fig. 1A,B). As in wild-type embryos, the first cardiac contractions in ded are peristaltic. By $36 \mathrm{~h}$ post-fertilization (hpf), the two chambers contract rhythmically, sequentially, and vigorously. However, shortly after, there is progressive diminution in ventricular contractility of ded mutant embryos. In order to quantitate contractility, we applied a fractional shortening assay, which measures systolic contractile function normalized to the diameters of the heart. By 48 hpf, fractional shortening (FS) of the ded ventricular chamber is severely reduced from $22.3 \% \pm 5.8 \%$ to $1.4 \% \pm 2.2 \%$. By $60 \mathrm{hpf}$ the ventricular chamber becomes completely silent, whereas the atrium of ded mutant embryos continues to contract normally (atrial FS of ded $=14.8 \% \pm 3.2 \%$; atrial FS of wild-type $=15.9 \% \pm 3 \%$ ) (Fig. 1C,D; Supplementary Movie 1). This indicates a chamber-specific requirement for ded to maintain cardiac ventricular contractility.

ded mutant ventricles are structurally indistinguishable from wild-type hearts. By $72 \mathrm{hpf}$, an epicardial, myocardial, as well as an endocardial layer are clearly present (Fig. 1E,F). The ventricular myocardium of ded thickens properly by addition of myocardial cells, and the number of ventricular cardiomyocytes ( $342 \pm 21$ SEM) is indistinguishable from wild type ( $336 \pm 34$ SEM), even at $72 \mathrm{hpf}$, when the ded ventricle is completely silent. There is no
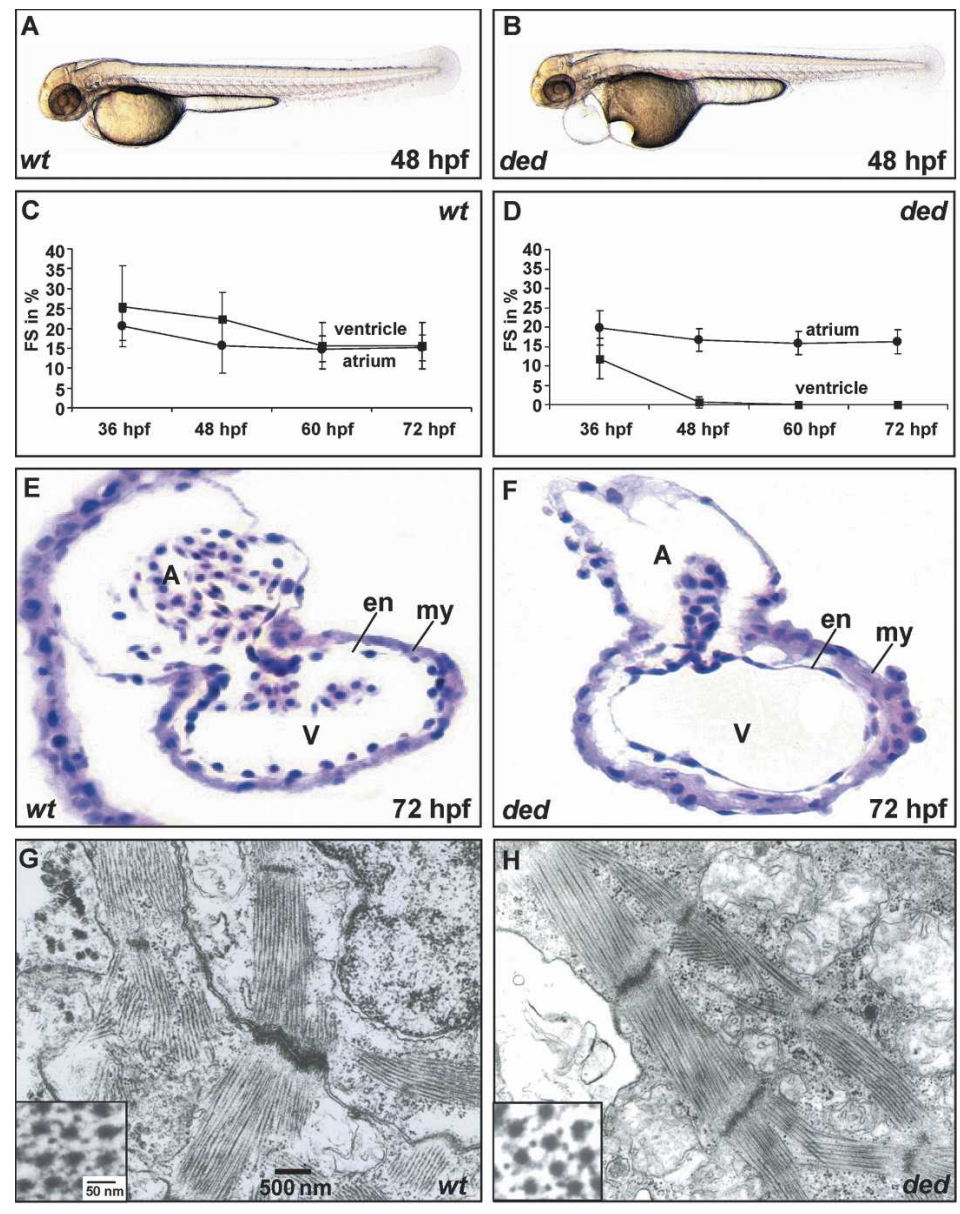

Figure 1. Effects of the ded $d^{m 58}$ mutation on embryonic heart function. $(A, B)$ ded mutants develop pericardial edema due to loss of ventricular contractility, whereas the development of other organ systems proceeds normally. Lateral view of wild-type (wt) and ded mutant embryos at $48 \mathrm{hpf}$. $(C, D)$ Fractional shortening (FS) of the atrial (filled circles) and ventricular (filled boxes) chamber of wild-type and ded mutants at 36,48 , 60 , and $72 \mathrm{hpf}$. Ventricular FS of ded mutant ventricles severely decreases over time, whereas atrial contractility is unaffected. $(E, F)$ ded hearts display normal heart morphology. Endocardial and myocardial layers of the heart are unaltered in ded mutant hearts after $72 \mathrm{hpf}$. Transverse sections of a wild-type $(E)$ and ded mutant $(F)$ embryo stained with hematoxylin/eosin. (A) Atrium; (V) ventricle; (en) endocard; (my) myocard. $(G, H)$ Transmission electron microscopy of wild-type $(G)$ and ded mutant $(H)$ zebrafish embryonic hearts at 72 hpf. Ventricular cardiomyocytes of ded mutants show normal cell architecture. Insets display transverse sections of myofilaments. 
increase in number of apoptotic myocardial cells compared with wild-type embryos using TUNEL-staining (data not shown). Transmission electron microscopy of ded ventricular cardiomyocytes reveals normal myocardial cell architecture, including regular arrays of thick and thin myofilaments, Z-discs, and intercalated discs, as well as normal content and appearance of mitochondria (Fig. 1G,H). There is no evident difference in the expression of key-regulatory cardiac transcription factors, such as znkx2.5 (Chen and Fishman 1996) and ztbx5 (Garrity et al. 2002), nor for genes encoding thick myofilament components, such as myosin light chain 2 (zmlc2), ventricular-specific myosin heavy chain (zvmhc) (Yelon et al. 1999) and atrial myosin heavy chain (zamhc) (Fig. 2A,F; Berdougo et al. 2003).

ded mutant embryos are also defective in embryonic vasculogenesis, lacking a lumenized dorsal aorta and the posterior cardinal vein (Fig. 2G,H).

dead beat acts cell autonomously in the cardiac myocyte

To evaluate whether ded acts cell autonomously or noncell autonomously in the ventricular myocardium, we generated fish with hearts mosaic between wild-type and ded mutant cardiomyocytes. Labeled wild-type cells integrate normally in ded $^{-/-}$ventricles and contract actively $(n=17)$ in the ded ventricle, even long after ded ventricular cardiomyocytes stopped contracting (Supplementary Movie 2). Myocytes from $\mathrm{ded}^{-/-}$mutant embryos in ventricles of wild-type embryos do not contract. Rather, they buldge outward during ventricular systole $(n=4)$ (data not shown). These experiments clearly indicate a ventricle-specific and cell-autonomous role of ded in the heart.

The dead beat $\left(\mathrm{ded}^{\mathrm{m} 582}\right)$ locus encodes zebrafish phospholipase $C \gamma 1$ (zplc $\gamma 1$ )

We identified the ENU-induced mutation causing the recessive ded mutant phenotype by a positional walk (Fig. 2I). The ded mutation maps to the zplcy1 gene. By sequence, we identified the ded ${ }^{\text {m582 }}$ mutation to be a guanine to thymine nucleotide transversion (GGA $\rightarrow$ TGA) in codon 11 , predicted to change glycine to a stop codon at amino acid 11 , and to cause premature termination of translation of $z p l c \gamma 1$ (Fig. 2J). Hence, the ded mutant

Figure 2. ded mutant heart and vascular phenotype due to mutation in the zebrafish phospholipase $C \gamma 1$ gene. $(A-F)$ Unaltered expression of heart chamber-specific genes in ded mutant hearts. At $48 \mathrm{hpf}$, the expression of myosin light chain 2 (mlc2), ventricular myosin heavy chain (vmhc), and atrial myosin heavy chain $(a m h c)$ investigated by RNA antisense in situ hybridization, is similar in ded and wild-type hearts. $(G, H)$ Absence of a lumenized dorsal aorta and posterior cardinal vein in ded mutants. Transverse sections of a wild-type $(G)$ and ded mutant $(H)$ embryo at the midtrunk region, stained with hematoxylin/eosin. (noto) Notochord; (GT) gut tube; (DA) dorsal aorta; (PCV) posterior cardinal vein. $(I)$ Integrated genetic and physical map of the zebrafish ded region. The ded mutation interval is flanked by the microsatellite markers z8945 and z44679. A bacterial artificial chromosome (BAC) clone SJ-BAC12 was isolated covering the ded interval and subjected to shotgun sequencing. Sequence analysis of the ded mutation interval revealed two zebrafish genes, highly homologous to the DNA topoisomerase I and phospholipase $C \gamma 1$. The genomic structure of zplcy1 is displayed at the bottom of the figure. The ded nonsense mutation $(\mathrm{G} \rightarrow \mathrm{T})$ in the first exon of $z p l c \gamma 1$ is indicated. $(J)$ The point mutation $(\mathrm{G} \rightarrow \mathrm{T})$ at cDNA position 31 translates into a premature stop codon (GGA $\rightarrow$ TGA). The mutated base is marked by an arrowhead.

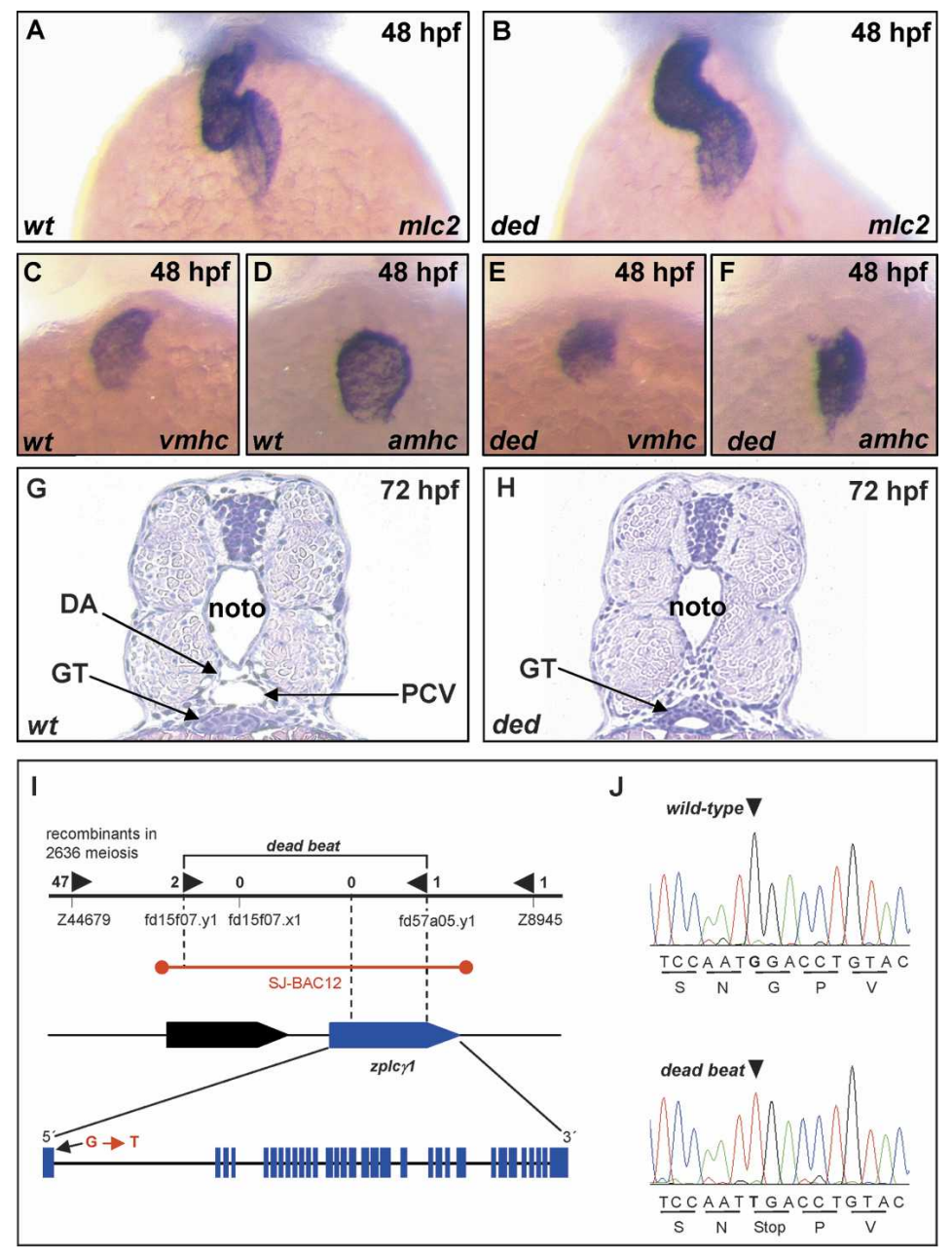


heart and vascular phenotypes are due to a loss of zplcy1 function.

As is the human, zplcy 1 is encoded by 32 exons. The zebrafish protein encodes 1312 amino acids and has $74 \%$ amino acid identity to the human ortholog. As in other species, zplcy1 encodes a pleckstrin homology domain (PH domain) in the $\mathrm{N}$ terminus of the protein, two catalytic domains ( $\mathrm{X}$ and $\mathrm{Y})$, and a conserved $\mathrm{C} 2$ domain in the C-terminal region. In addition, PLC $\gamma 1$ includes two $\mathrm{SH} 2$ domains, known to recognize the phosphotyrosinecontaining sequences in VEGF receptors, one SH3 domain, and one EF-hand domain (Supplementary Fig. S1A; Anderson et al. 1990).

In other species, plcy 1 is expressed ubiquitously both during embryonic development and in the adult (Gerfen et al. 1988; Liao et al. 2002). We confirm here by RTPCR expression of zplcy1 in the myocardium of both atrium and ventricle during embryonic development and in the adult vertebrate (Supplementary Fig. S1B). By antisense RNA whole-mount in situ staining, we observe zplcy 1 to be ubiquitously expressed in zebrafish embryos during the first $72 \mathrm{hpf}$. At $48 \mathrm{hpf}$, expression of $z p l c \gamma 1$
RNA is pronounced in the brain, vasculature, and the heart (Fig. 3A-C). By $72 \mathrm{hpf}, \mathrm{zPLC} \gamma 1$ protein is found to be expressed in both the endocardial and myocardial layer of the atrium and the ventricle (Fig. 3D).

\section{Injection of Morpholino-modified antisense oligonucleotides against zebrafish plc 1 phenocopies the ded heart and vessel phenotypes}

To confirm that the ded mutant heart contractility and vascular phenotypes are caused by the absence of zPLC $\gamma 1$, we injected Morpholino-modified antisense oligonucleotides, either directed against the translational start site (MO1-zplcy1) or the splice donor site of exon 13 (MO2-zplcy1) of zplcy1 into one-cell stage wild-type zebrafish embryos. When injected with $4 \mathrm{ng}$ of MO2zplc $1,98 \%$ of injected embryos reveal the ded mutant heart and vascular phenotype (Fig. 3E-G). Blood circulation in lumenized vessels is absent, and ventricular contractility decreases during development, leading to a silent ventricle $60 \mathrm{~h}$ after injection. Similar effects and efficacy are observed when injecting $4 \mathrm{ng}$ of MO1-zplcy1.
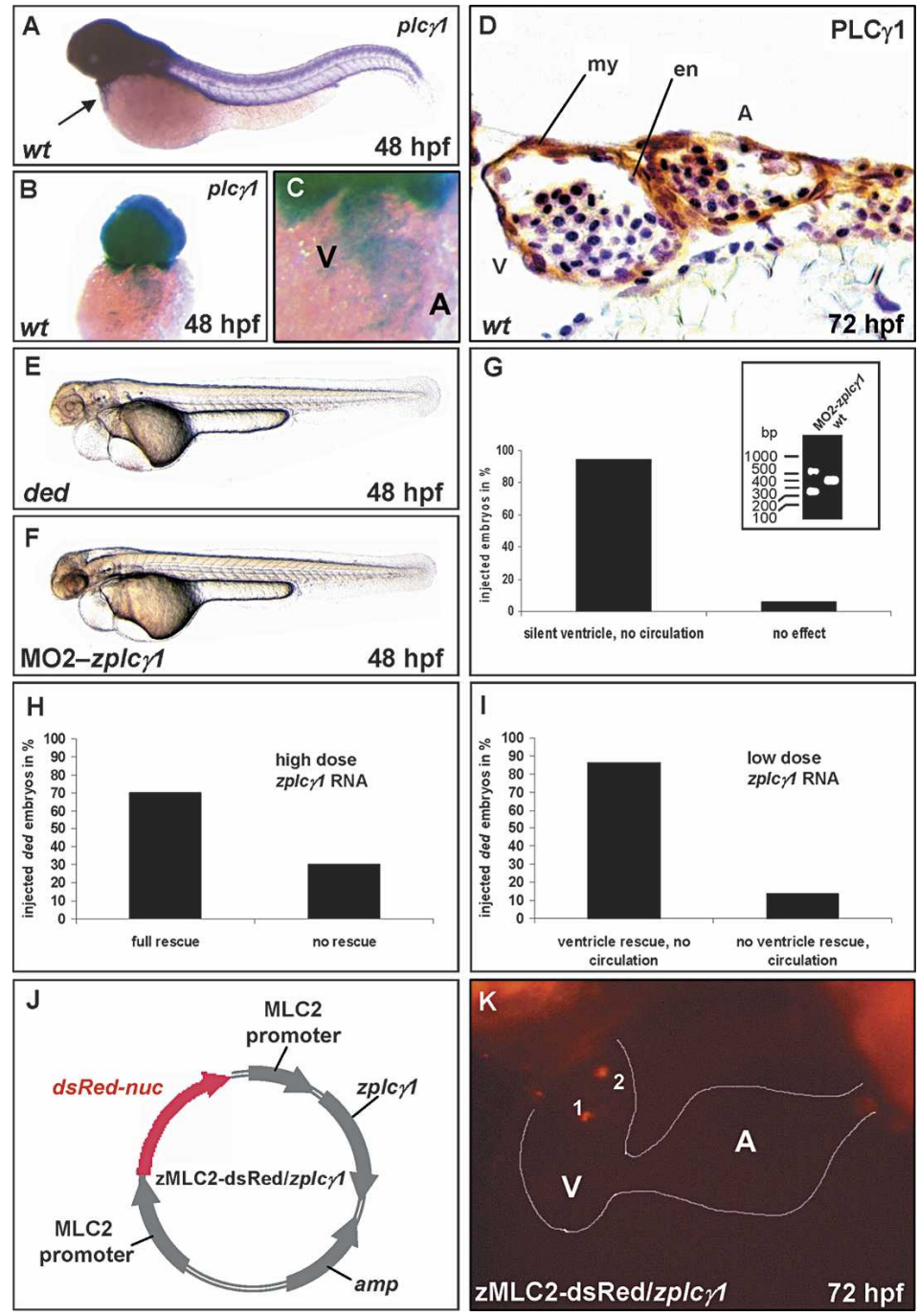

Figure 3. The ded ventricular contractility defect can be rescued even in the absence of an intact vasculature. $(A-D)$ Ubiquitous zplcy1 expression during zebrafish development. $(A-C)$ By 72 hpf zplcy1, RNA expression is pronounced in the brain, vasculature, and the heart (arrow). (D) Immunostain of wild-type zebrafish embryos using a PLC $\gamma 1$-specific antibody. PLC $\gamma 1$ is expressed in the endocardial and myocardial layer of both the atrium and the ventricle. (A) Atrium; (V) ventricle; (en) endocardium; (my) myocardium. (E-G) Inhibition of zplcy1 function by Morpholino-modified antisense oligonucleotide injection phenocopies the ded mutant phenotype. MO2-zplcy1-injected embryos are indistinguishable from ded mutant embryos and display severe impairment of ventricular contractility and vasculogenesis. The effectiveness of MO2-zplcy1 on mRNA splicing is shown in the inset in G. Injection of MO2-zplc 1 results in abnormal splice products of 602 bp (integration of intron 13) and $250 \mathrm{bp}$ (skipping of exon 13), leading to premature termination of translation of zPLC $\gamma 1$. $(H, I)$ Injection of wild-type zplcy1 RNA at different dosages rescues the ded mutant vascular and heart defects, independently. $(H)$ Injection of zplcy1 mRNA at a high dose $(25 \mathrm{pg})$ results in the full rescue of the ded mutant vascular and heart phenotype in $70 \%$ of injected $\mathrm{ded}^{-/-}$ embryos. (I) If lower amounts (13 pg) of zplcy1 mRNA are injected in $\mathrm{ded}^{-1-}$ embryos, $\sim 80 \%$ of injected embryos reveal normal ventricular contractility after 72 hpf, whereas no vascular rescue was observed. Vice versa, $\sim 15 \%$ of rescued $\mathrm{ded}^{-1-}$ embryos show complete vascular rescue, whereas ventricular contractility is still absent. $(J, K)$ Cardiac-specific, mosaic overexpression of wild-type zplc 1 rescues the contractile deficit of single $\mathrm{ded}^{-/-}$cardiomyocytes. (J) Schematogram of the $z$ MLC2-dsRed/zplcy1 vector. $(K)$ Injection of zMLC2-dsRed/zplc 1 leads to cardiac-specific, mosaic overexpression of zplcy1 in two different cardiomyocytes $(1,2)$. (Lateral view) Head to the left. (V) Ventricle; (A) atrium. 
RNA analysis confirms the effect of MO2-zplcy1 on zplcy1 RNA (Fig. 3G, inset).

zplc $1 \mathrm{mRNA}$ independently restores heart function and vasculogenesis in ded mutant embryos

To evaluate whether wild-type $z p l c \gamma 1$ mRNA can restore ventricular contractile function and/or vasculogenesis of homozygous ded mutant embryos, we injected zplcy 1 mRNA into embryos derived from intercrossing ded heterozygous zebrafish. After injecting 25 pg of wildtype plcy 1 mRNA, $70 \%$ of ded mutant embryos show complete rescue of both the vascular and heart phenotype (Fig. 3H). Blood circulation through a lumenized arterial and venous vasculature is established and the ventricle contracts normally. Cardiac contractility is generally restored by lower amounts than needed to rescue the vasculature. In $86.3 \%$ ded $^{-/-}$embryos injected with 13 pg of zplcy1 mRNA, ventricular heart contractility is restored, but no blood circulation is established. In a few cases $(13.6 \%)$, blood vessels are restored, but the ventricular chamber does not pump (Fig. 3I). Presumably, this reflects a mosaic effect of the injection. These results confirm that the mutation in the zplcy 1 gene is responsible for the ded vascular and heart defects. Furthermore, they demonstrate that the cardiac phenotype is not secondary to the vascular defect.

Cardiac-specific, mosaic overexpression of zebrafish plc $\gamma 1$ rescues the ded mutant ventricular contractility defect

In order to examine whether zplcy1 is sufficient to rescue ventricular cardiomyocyte contractility in a cell-autonomous fashion, we directed its expression to myocardial cells of the heart using the cardiomyocyte-specific zebrafish myosin-light chain 2 (zMLC2) promoter (Rottbauer et al. 2002). We modified our previous expression construct, such that the fluorescent marker nucDsRed2 is coexpressed (zMLC2-dsRed/zplcy1) (Fig. 3J). We injected the zMLC2-dsRed/zplcy1 plasmid into $\mathrm{ded}^{-/-}$embryos, generating mosaic hearts. Cardiomyocytes, which express zebrafish zplc 1 and nucDsRed2 under the control of zMLC2 contract vigorously in otherwise silent ded ventricles (Fig. 3K; Supplementary Movie 3). This persists beyond $72 \mathrm{hpf}$, indicating that overexpression of wild-type $z p l c y 1$ in $\mathrm{ded}^{-/-}$cardiomyocytes can restore the ded ventricular contractility defect in single ventricular cells (18 of 30 ).

VEGF is essential for zebrafish vasculogenesis and cardiac ventricular contractility

PLC $\gamma 1$ acts downstream of VEGF signaling in many situations (McLaughlin and De Vries 2001; Takahashi et al. 2001; Lawson et al. 2003). In zebrafish, knock-down of vegf using Morpholino-modified antisense oligonucleotides (MO-vegf) have been noted to cause a vascular phe- notype identical to the one observed in ded mutant embryos (Nasevicius et al. 2000), a finding we confirm.

In addition, we find that contractility of the ventricle is significantly impaired by Morpholinos directed to vegf. Fractional shortening of the ventricular chamber is reduced from wild-type values of $34.7 \% \pm 6.2 \%$ to $5.7 \% \pm 3.6 \%$ in injected embryos after 48 hpf. By 60 hpf, the ventricle in the Morpholino-injected embryos becomes silent, while the atrium continues to contract (Fig. 4C). Thus, MO-vegf injection phenocopies both the vascular and the cardiac phenotypes of ded mutant zebrafish embryos (Fig. 4A-D; Supplementary Movie 4), indicating that VEGF is critical to ventricular contractility.

VEGF signals through its receptor VEGFR-1 (FLT-1) to control ventricular contractility

On endothelial cells, where best studied, VEGF is known to exert its effects through at least two receptors, FLK-1, FLT-1, along with the coreceptor Neuropilin-1 (Np-1) (Neufeld et al. 1999; Gu et al. 2003). In zebrafish, only FLK-1 and Np-1 have been identified to date (Thompson et al. 1998; Lee et al. 2002). Therefore, to evaluate which receptor mediates VEGF effects on zebrafish ventricular contractility and vasculogenesis, we first inhibited zebrafish FLK-1 and Np-1 by injections of Morpholinos. However, none of them seems to perturb the heart. Np-1 Morpholino has no obvious effects (data not shown; Lee et al. 2002). As in flk-1 mutant embryos (Habeck et al. 2002), flk-1 Morpholino interferes selectively with formation of the intersegmental vessels (in $91.2 \%$ of injected embryos, as shown in Fig. 4E-H). Hence, neither FLK-1 nor Np-1 appear to be the relevant receptors for VEGF effects on cardiac contractility or vasculogenesis in the zebrafish.

In mammals and the chick (Yamaguchi et al. 2002), there is an additional VEGF receptor, termed FLT-1 or VEGFR-1. To our knowledge, a FLT-1 receptor has not been identified in zebrafish. In fact, it has been speculated that FLK-1 serves as the only VEGF receptor in that species, similar to the situation in Drosophila melanogaster (Habeck et al. 2002). However, since we found no evidence that FLK-1 or the coreceptor Np-1 mediate VEGF effects on cardiac contractility in the zebrafish, we suspected the existence of another, as yet undefined, zebrafish VEGF receptor. We identified in the zebrafish genome sequence an open reading frame with $51 \%$ amino acid identity to human FLT-1 (GenBank accession no. AY848694). We located this zebrafish flt-1 gene on zebrafish linkage group 24 by radiation hybrid mapping. Interestingly, the zebrafish genomic region around the flt-1 gene (NA54293 from Sanger Zebrafish Assembly 3) is syntenic to a region on human chromosome 13 that includes the flt-1 gene, further supporting the identification of zebrafish flt-1 as the ortholog of human flt-1 (data not shown). Full-length alignment of human and zebrafish FLT-1 protein sequence is provided as Supplemental Material (Supplementary Fig. S3). 


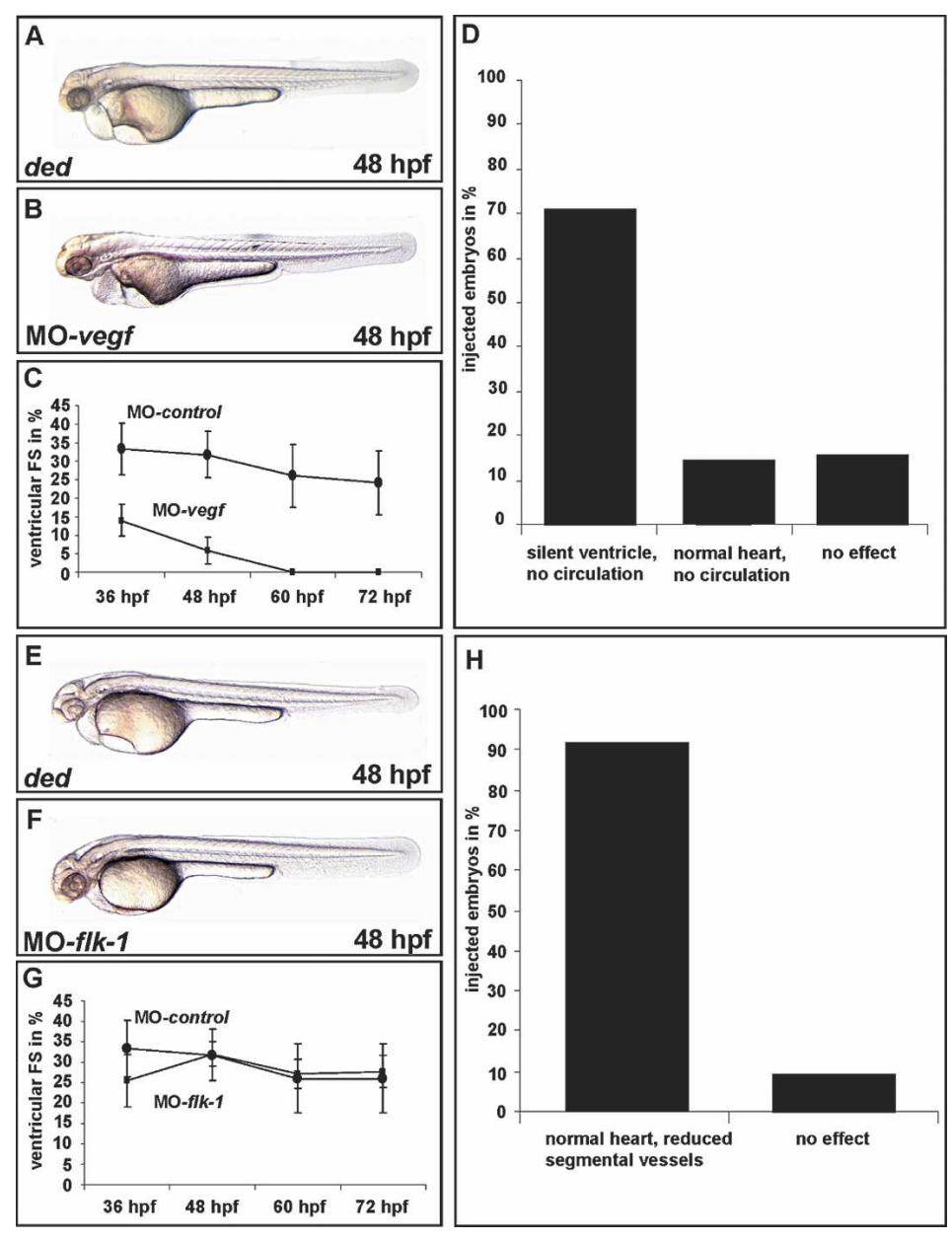

Figure 4. Effects of VEGF on zebrafish cardiac ventricular contractility are not mediated by the receptor FLK-1. $(A-D)$ Injection of MO-vegf into wild-type embryos phenocopies the vascular and heart contractility defect of ded mutant embryos. At $60 \mathrm{hpf}, 70 \%$ of injected embryos reveal complete absence of circulation, as well as a silent ventricle. About $15 \%$ of embryos reveal loss of circulation, but normal ventricular contractility. $(C)$ In contrast to mock-injected (MO-control) wild-type embryos (filled circles), fractional shortening of the ventricular chamber of MO-vegf-injected embryos (filled boxes) is significantly reduced after $36 \mathrm{hpf}$. By $60 \mathrm{hpf}$, the ventricle of MO-vegf-injected embryos becomes silent $(\mathrm{FS}=0 \%)$. $(E-H)$ Zebrafish embryos injected with MO-flk-1 $(F)$ do not display a vascular and cardiac phenotype similar to ded mutants $(E)$. $(G)$ In comparison with mock-injected (MO-control) wild-type embryos (filled circles) injection of MO-flk-1 (filled boxes) does not lead to a decreased fractional shortening (FS) of the ventricular chamber. (H) In $\sim 90 \%$ of MO-flk1 -injected embryos, intersegmental vessels are absent. None of the embryos displays a heart phenotype similar to ded mutant embryos.
To test whether zebrafish flt-1 transduces VEGF signals, we injected embryos with zebrafish flt-1 Morpholino (MO1-flt-1) (Fig. 5A-C). Eighty percent of injected wild-type embryos phenocopy the vascular phenotype of ded mutant embryos (data not shown). In addition, MO1-flt-1 injection causes progressive reduction of ventricular contractility to a fractional shortening of $4.8 \% \pm 5 \%$ by $48 \mathrm{hpf}$, and finally between 60 and $72 \mathrm{hpf}$, to a silent ventricle (Fig. 5C; Supplementary Movie 4). This is identical to the cardiac phenotype observed in ded mutant and MO-vegf-injected zebrafish embryos. As in ded mutant embryos, the heart in MO1-flt-1-injected embryos retain relatively normal histology of endocardial and myocardial layers (Fig. 5D). Ventricular cardiomyocyte cell numbers are not different between MO1flt-1 (325 \pm 18 SEM) and embryos injected with MO-control $(341 \pm 21 \mathrm{SEM})$ at $72 \mathrm{hpf}$. Injection of a second zebrafish flt-1 Morpholino (MO2-flt-1), which targets a different splice donor site, also phenocopies the ded mutation with the same efficacy. RT-PCR analysis demonstrates strong expression of flt-1 in embryonic and adult cardiomyocytes from zebrafish and rat (Supplementary Fig. S2A). In summary, the data indicate that VEGF signaling, mainly through the VEGF receptor 1 (FLT-1), activates zPLC $\gamma 1$, and thereby controls ventricular contractility.

\section{VEGF receptors and $P L C \gamma 1$ are regulators of rat} cardiomyocyte contractility

VEGF and PLC $\gamma 1$ are known to be expressed in cardiomyocytes (Puceat and Vassort 1996; Seko et al. 1999), whereas FLT-1 expression was thought to be mainly on vascular endothelial cells (Shibuya et al. 1994). We therefore evaluated the expression of FLT-1 protein in adult rat hearts by immunostaining. As shown in Figure 5E, FLT-1 is expressed at high levels in the sarcolemma of rat cardiomyocytes. An isotype-matched antibody control does not show any specific immunostaining in the sarcolemma (Supplementary Fig. S2B).

To examine whether VEGF signaling through the receptor tyrosine kinase FLT-1 and the consecutive activation of PLC $\gamma 1$ affect cardiac myocyte contractility in mammals, we first incubated neonatal rat ventricular cardiomyocytes with the specific VEGF receptor inhibitor PTK787/ZK222584 or the PLC inhibitor U73122 at various concentrations and recorded their spontaneous contractility by video-microscopy (Yule and Williams 1992; Wood et al. 2000). Eighty-four percent of cardiomyocytes treated with PTK787/ZK222584 lose their spontaneous contractile activity within minutes after incubation. Similarly, up to $80 \%$ of ventricular rat cardio- 
Rottbauer et al.

Figure 5. VEGF-FLT-1-PLC $\gamma 1$ signaling controls cardiomyocyte contractility by modulating calcium cycling. $(A-C)$ MO1-flt-1-injected embryos $(B)$ display a cardiovascular phenotype indistinguishable from ded mutant embryos $(A)$. (C) Injection of MO1-flt-1 significantly impairs cardiac ventricular contractility. In contrast to MO-control-injected embryos (filled circles), fractional shortening of the ventricular chamber of MO1-flt-1-injected embryos (filled boxes) is significantly reduced after $36 \mathrm{hpf}$. By $60 \mathrm{hpf}$, the ventricle of MO1-flt-1-injected embryos becomes silent ( $\mathrm{FS}=0 \%$ ). (D) Histological sections of MO1-flt-1-injected zebrafish embryonic hearts at $72 \mathrm{hpf}$ stained with hematoxylin/eosin. The ventricular and atrial myocardium of MO1-flt-1-injected embryos is properly developed by $72 \mathrm{hpf}$. Both heart chambers display normal myocardial and endocardial layers. (A) Atrium; (V) ventricle; (en) endocard; (my) myocard. (E) The VEGF-1 receptor FLT-1 is expressed in the cell membrane of rat ventricular cardiomyocytes. Cell nuclei are counterstained with hematoxylin. $(F) \mathrm{Ca}^{2+}$ transients of neonatal rat ventricular cardiomyocytes are modified by VEGF-PLC $\gamma 1$ signaling. $\mathrm{Ca}^{2+}$ transients (Fura-2 F340/F380) were measured before and $3 \mathrm{~min}$ after treatment with the PLC inhibitor U73122, the VEGF receptor inhibitor PTK787/ZK222584 or VEGF 164 . Cardiomyocytes were field stimulated at a rate of $2 \mathrm{~Hz}$. Inhibition of PLC $\gamma 1$ function by U73122 (1 $\mu \mathrm{M})$ or VEGF receptor function by PTK787/ZK222584 (5 $\mu \mathrm{M}$ ) leads to significant reduction of the calcium transients, whereas treatment with $\mathrm{VEGF}_{164}$ leads to significant increase of calcium cycling.
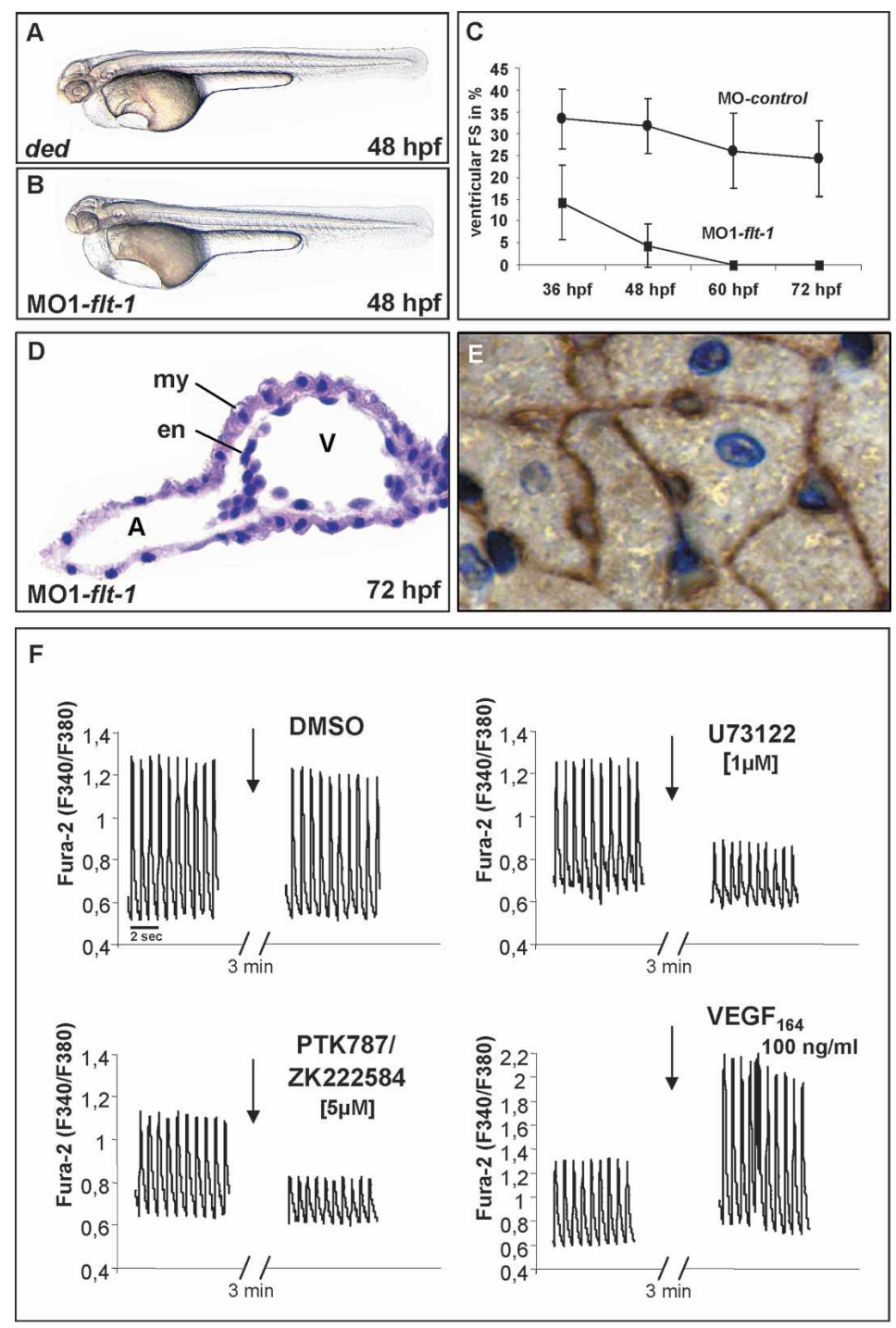

myocytes treated with the PLC inhibitor U73122 lose their spontaneous contractile activity within minutes after incubation, dependent on the amount of U73122 used $(10-50 \mu \mathrm{M})$, whereas cardiomyocytes treated with the inactive form of the inhibitor (U73343) continue beating. PTK787/ZK222584 and U73122 treated cardiomyocytes are refractory to electrical pacing. These findings already indicate an essential role for the VEGF-PLC $\gamma 1$ pathway in mammalian ventricular cardiomyocyte contractility.

To further elucidate the mechanism by which VEGFFLT-1-PLC $\gamma 1$ signaling controls cardiomyocyte contractility, we paced neonatal rat ventricular cardiomyocytes at a defined rate (two per second), incubated them with the specific VEGF receptor inhibitor PTK787/ZK222584, the PLC inhibitor U73122 as well as $\operatorname{VEGF}_{164}$ at various concentrations, and recorded their calcium transients before and after treatment. As shown in Figure 5F, inhibition of VEGF signaling with either PTK787/ZK222584 $(5 \mu \mathrm{M})$ or U73122 $(1 \mu \mathrm{M})$ significantly reduces $(-20 \%-$ $30 \%$ ) calcium transients in the ventricular cardiomyo- cytes, whereas treatment with $\operatorname{VEGF}_{164}(100 \mathrm{ng} / \mathrm{mL})$ almost doubles calcium transients. Treatment of the fieldstimulated cardiomyocytes with high concentrations of PTK787/ZK222584 $(20 \mu \mathrm{M})$ or U73122 $(20 \mu \mathrm{M})$ completely inhibits calcium cycling within 3 min after incubation (data not shown). These findings indicate that VEGF-PLC $\gamma 1$ signaling controls mammalian ventricular cardiomyocyte contractility by modulating calcium cycling.

Interestingly, when bathing wild-type zebrafish embryos in either U73122 (1 $\mu \mathrm{M})$ or PTK787/ZK222584 $(5 \mu \mathrm{M})$ after $48 \mathrm{~h}$ of development, at a time point when both heart chambers are usually vigorously beating and propel blood cells to the aterial and venous vascular system, ventricular cardiomyocytes become silent within $24 \mathrm{~h}$, whereas no effect on the vascular system is observed (Supplementary Movie 5). These findings are compatible with the VEGF-FLT-1-PLC $\gamma 1$ signaling pathway, playing an essential role in controlling ventricular cardiomyocyte contractility, even in later stages of development. 


\section{Discussion}

Cardiac contractility is regulated tightly as an essential homeostatic mechanism (Starling 1918; Frank 1959; Schaldach and Hutten 1990). Some of the control is neuro-humoral, but much appears intrinsic to the heart. Here we show that VEGF, in addition to its role in bloodvessel formation, regulates cardiac contractility. Its pathway of action specifically uses the FLT-1 receptor and PLC $\gamma 1$.

We describe here a mutation in zebrafish plc 1 , dead beat, which impairs proper arterial and venous vasculogenesis, as well as cardiac ventricular contractility. The mutation completely abolishes plc $\gamma 1$ function. PLC $\gamma 1$ is known to act downstream of VEGF to control arterial vasculogenesis in vertebrates (Liao et al. 2002; Lawson et al. 2003). We find here that PLC $\gamma 1$ also is essential for vertebrate heart contractility. Antisense-oligonucleotide mediated knock-down of either vegf, flt-1, or plc 1 function in zebrafish leads to a vascular and cardiac phenotype identical to ded. Injected embryos lack a lumenized arterial and venous vascular system, and cardiac ventricular contractility declines over time to culminate in a silent heart by $60 \mathrm{hpf}$. All components of the pathway, namely VEGF, its receptor FLT-1, and PLC $\gamma 1$, are found to be expressed in vertebrate cardiomyocytes. Inhibition of VEGF signaling reduces calcium transients in neonatal rat ventricular cardiomyocytes, whereas exposure to VEGF leads to increased calcium cycling. Therefore, we postulate that VEGF activates PLC $\gamma 1$ in ventricular cardiomyocytes, specifically through its receptor FLT-1 to control their contractile force.

Molecular pathways essential for both vasculogenesis and heart development are difficult to dissect genetically in placental animals, because disturbance of either vascular or heart development leads to early embryonic death. Nullizygous vegf, flt-1,flk-1, and plc 1 mice die in early embryogenesis due to disturbed vasculogenesis and angiogenesis (Shalaby et al. 1995; Carmeliet et al. 1996; Ferrara 2001; Liao et al. 2002). In contrast to mammals, intact cardiovascular function is not essential for the early development of the zebrafish embryo, because it obtains adequate oxygen by diffusion from its environment (Burggren and Pinder 1991; Stainier and Fishman 1994). Zebrafish embryos, mutant in cardiovascular gene function, survive for days to late larval stages. This permits analysis of the role of zPLC $\gamma 1$ in the development of cardiac function.

The zPLC $\gamma 1$ effect is cell-autonomous. Cardiac myocytes express zPLC $\gamma 1$. Cardiac myocytes without zPLC $\gamma 1$ do not contract even in a wild-type heart, whereas wild-type cardiac myocytes contract in a $\mathrm{ded}^{-/-}$ heart. The contractile deficit of $\mathrm{ded}^{-/-}$ventricular cardiomyocytes can be rescued by cardiac-specific overexpression of $z p l c \gamma 1$, even in single ventricular cardiomyocytes that are surrounded by noncontracting cardiac cells.

Although zplcy1 is found to be expressed in atrial cardiomyocytes, it seems not to be essential for maintaining atrial cardiomyocyte contractility. The atrium in ded mutant embryos continues to contract normally, even long after the ventricle becomes silent. The biology of the myocytes in the two chambers is quite distinct. For example, the $\alpha 1 \mathrm{C}$ L-type calcium channel subunit (C-LTCC) is expressed in both the atrium and the ventricle of the zebrafish heart, but its mutation only affects the ventricle (Rottbauer et al. 2001).

The downstream mediator of PLC $\gamma 1$ in cardiomyocytes remains to be determined. Activated PLC $\gamma 1$ enzyme catalyses the hydrolysis of phosphatidylinositol 4,5,-bisphosphate $\left(\mathrm{PIP}_{2}\right)$ to the second messenger molecules inositol 1,4,5-triphosphate $\left(\mathrm{IP}_{3}\right)$ and diacylglycerol (DAG). $\mathrm{IP}_{3}$ can cause release of $\mathrm{Ca}^{2+}$ from internal stores, and thereby increase cardiomyocyte contractility. DAG activates PKC enzymes. However, the only PKC evaluated in cardiac myocytes to date, PKC- $\alpha$, appears to inhibit contractility (Braz et al. 2004). Other isoenzymes, such as PKC- $\delta$ and PKC- $\varepsilon$ might, of course, play different roles (Kang and Walker 2005).

VEGF is known to be produced and secreted by cardiomyocytes upon biomechanical stress (Li et al. 1997; Seko et al. 1999). It clearly is needed for cardiac vascularization, and it makes sense that vessel density might be a response to stress. In zebrafish, we find that a separate role for VEGF in controlling of heart contractility can be demonstrated long before intramyocardial capillaries and coronary arteries are generated (Hu et al. 2000). Therefore, VEGF signaling is not only required for proper arterial and venous vasculogenesis, but also for the maintenance of cardiac ventricular contractility.

In mammals, VEGF is known to exert its effects on endothelial cells through the receptors FLK-1 and FLT-1. FLK-1 has an essential role in vasculogenesis, angiogenesis, and hematopoiesis, and FLK-1 null mice do not establish the framework of a vascular system nor develop blood islands or organized blood vessels (Shalaby et al. 1995). FLK-1-deficient zebrafish embryos, on the other hand (Habeck et al. 2002), manifest more subtle effects on vessel formation, limited to deficiency in angiogenic formation of intersegmental vessels. This suggests the role of another VEGF receptor with regard to vasculogenesis in the zebrafish. As shown here, MO-flk-1 injections, even at high concentrations, do not affect heart function. We identified and characterized here the zebrafish orthologous gene for the VEGF receptor flt-1. The zebrafish orthologous FLT-1 sequence shows high homology to the human FLT-1 protein $(-51 \%$ amino acid identity) and maps to a zebrafish linkage group in a region syntenic to human chromosome 13, where human flt-1 is encoded. It is this receptor that appears to mediate VEGF effects on both cardiac contractility and vasculogenesis in the zebrafish.

The heart responds to stretch by increasing contractility. This feature allows it to respond to increased load, for example, as imposed by heightened blood pressure. Clinically, it is this response that underpins much of the compensatory response in the face of early cardiac failure from myocardial ischemia (Starling 1918; Frank 1959). The molecular basis for this phenomenon has been elusive. There is evidence that VEGF secretion increases 
with physical stretch of the heart, via glycoprotein gp 130 and transforming growth factor (TGF) $\beta$ (Seko et al. 1999; Funamoto et al. 2000). PLC $\gamma 1$ itself can be activated through mechanical stress in neonatal rat cardiomyocytes (Seko et al. 1998). Hence, it is conceivable that stretch of the heart, by increasing VEGF, could contribute to enhanced cardiac contractiltity.

Nearly 5 million Americans have heart failure today, and 550,000 new cases are diagnosed each year. The use of medicines that improve contractility by changing $\mathrm{Ca}^{2+}$ handling are complicated by concomitant propensity to arrhythmias. No current agents safely enhance cardiac contractility. It will be of interest to examine whether the VEGF-PLC $\gamma 1$ pathway may offer new opportunities for such intervention.

\section{Materials and methods}

\section{Zebrafish strains, compound treatment, and cell transplantation}

Care and breeding of zebrafish Danio rerio was as described (Westerfield 1995). Zebrafish embryos were incubated at different developmental stages with various concentrations of the VEGF receptor-specific inhibitor PTK787/ZK222584 (Schering) or the PLC inhibitor U73122 (Sigma). Pictures and movies were recorded 24,48 , and $72 \mathrm{~h}$ after starting the compound treatment.

Embryos used for transplantation experiments were produced by intercrossing $\mathrm{ded}^{\mathrm{m582-/+}}$ fish. Cell transplantation was performed essentially as described (Rottbauer et al. 2001). By 24, 48 , and $72 \mathrm{hpf}$, embryos were inspected with fluorescence microscopy, and movies of integrated ventricular cells recorded. To inhibit pigmentation, $0.003 \%$ 1-phenyl-2-thiourea was added to the embryo medium. To confirm the integration of labeled donor cells into ded or wild-type ventricular myocardial layers, whole embryos were stained with antibiotin antibodies, fixed, and sectioned. Labeled donor embryos were genotyped using polymorphic markers, flanking the ded gene.

Histology, transmission electron microscopy, RNA antisense in situ hybridization, and immunostaining

Embryos were fixed in 4\% paraformaldehyde and embedded in JB-4 (Polysciences, Inc.). Five-micrometer sections were cut, dried, and stained with hematoxylin/eosin. Electron Micrographs were obtained essentially as described (Rottbauer et al. 2001).

Whole-mount RNA in situ hybridization was carried out essentially as described (Jowett and Lettice 1994) using a fulllength $z p l c \gamma 1$ antisense probe, as well as antisense probes for zflk-1, zmlc2, zvmhc, zamhc, ztbx5, and znkx2.5. All RNA probes were digoxigenin labeled.

For immunostaining, adult rat hearts were fixed in $4 \%$ formalin and paraffin embedded. FLT-1 immunostaining was performed with the polyclonal anti-VEGF receptor 1 (ab2350, Abcam) and HRP-coupled rabbit IgG (ab6721, Abcam) antibodies. $\mathrm{DAB}$ staining was carried out according to the manufacturer's protocol (Sigma) and cell nuclei counterstained with hematoxylin. For immunostaining of Dent's fixed zebrafish embryos, the monoclonal anti-PLC $\gamma 1$ (Upstate) antibody and the Vectastain $\mathrm{ABC}$ kit (VectorLabs) was used. Embryos were embedded in JB-4 (Polysciences, Inc.), 5- $\mu$ m sections were cut and dried, and cell nuclei were counterstained with hematoxylin.
Genetic mapping, positional cloning, and mutation detection

DNA from 24 ded $^{m 582}$ mutant and 24 wild-type embryos was pooled and bulked segregation analysis performed as described (Michelmore et al. 1991). The critical genomic interval for ded was defined by genotyping 1318 mutant embryos for polymorphic markers in the area. RNA from ded mutant and wild-type embryos was isolated using TRIZOL Reagent (Life Technologies) and reverse transcribed. Eight independent clones from mutant and wild-type zplcy 1 cDNA were sequenced. Genomic DNA from ded mutant and wild-type embryos was sequenced around the point mutation.

\section{Injection procedures}

Morpholino-modified oligonucleotides were directed against a splice donor site (MO2-zplcy1, 5'-AGAGCGTCCTCCTGA CCTTGATGAG-3') and the translational start site of $z p l c \gamma 1$ (MO1-zplcy1, 5'-TCGCAGCCATTTCCCCTGTTTGTTC-3'), against the translational start site of vegf (MO-vegf, 5'-GTAT CAAATAAACAACCAAGTTCAT-3') (Nasevicius et al. 2000), against the 5'UTRs of $f 1 k-1$ (MO-flk-1, 5'-CCGAATGATACTC CGTATGTCAC-3') and neuropilin-1 (MO-np-1, 5'-GAATCCT GGAGTTCGGAGTGCGGAA-3'), and two different splice donor sites of $z f l t$-1 (MO1-flt-1, 5' -CAGCAGTTCACTCACATCT CCGTTC-3') and (MO2-flt-1, 5'-AAGCAGACTCTCACCATT TACCACC-3'). A standard control oligonucleotide (MO-control) (GENETOOLS, LLC) was injected at the same concentration as a negative control.

To drive cardiac-specific expression of $z p l c \gamma 1$, the coding sequence was cloned downstream of the zebrafish MLC2 promoter (zMLC2-dsRed- zply1). Seventy-five picograms of zMLC2-dsRed- zply1 were pressure injected into one-cell stage wild-type and mutant embryos produced by intercrossing ded $^{m 582-/+}$ fish. zplcy1-expressing cells were identified in vivo by fluorescent video microscopy (EM CCD Camera Proxitronic).

Sense-capped RNA was synthesized using the mMESSAGE mMASCHINE system (Ambion) from pCS2zplcy1. RNA was diluted $(26 \mathrm{ng} / \mu \mathrm{L}$ or $50 \mathrm{ng} / \mu \mathrm{L}$ in solution A $[0.1 \%$ phenol red, $0.2 \mathrm{M} \mathrm{KCl}])$ and microinjected into one-cell stage embryos. Siblings from the same pool were injected using solution A as control.

\section{Cardiomyocyte culture and measurement of $\mathrm{Ca}^{2+}$ transients}

Preparation and cultivation of neonatal and adult ventricular cardiomyocytes was performed essentially as described (Most et al. 2003). Adult as well as neonatal cardiomyocytes were incubated with different concentrations of the phospholipase C-specific inhibitor (U73122, Sigma) or its inactive form (U73343, Sigma). Futhermore cardiomyocytes were incubated with different concentrations of the VEGF receptor (FLT-1, FLK-1) inhibitor (PTK787/ZK222584, Schering).

$\mathrm{Ca}^{2+}$ imaging in field-stimulated neonatal ventricular cardiomyocytes was carried out essentially as described (Most et al. 2003). Isolated cells were incubated in $1 \mathrm{~mL}$ HEPES-modified medium $199\left(2 \mathrm{mM}\left[\mathrm{Ca}^{2+}\right]\right)$ with $2 \mu \mathrm{M}$ FURA2-AM for $20 \mathrm{~min}$ at room temperature. Cells were electrically stimulated with $2 \mathrm{~Hz}$, and measurements were performed with T.I.L.L. Vision software (version 4.01). $\mathrm{Ca}^{2+}$ transients were recorded at baseline and throughout the treatment with the VEGF receptor-specific inhibitor PTK787/ZK222584 (Schering), the PLC inhibitor U73122 (Sigma), and VEGF 164 (Sigma). 


\section{Acknowledgments}

We thank M.H. Boulos, M. McKee, S. Marquart, and M. Völckers for technical assistance, and A. Doherty for excellent fish care. We are especially grateful to Melanie Peters, who provided us excellent technical assistance and tragically died during the course of this study. We thank N.D. Lawson for providing pCS2zplcg1 and J.G. Burns supplying us zMLC2-dsRed. This work was supported by grants from NIH-5R01HL49579, 5R01DK55383, 1R01HL63206 (M.C.F.), DFG Ro2173/1-1, DFG Ro2173/2-1, and BMBF 01GS0108-TP5, as well as Klaus-Georg and Sigrid Hengstberger Stipendium (W.R.).

\section{References}

Anderson, D., Koch, C.A., Grey, L., Ellis, C., Moran, M.F., and Pawson, T. 1990. Binding of SH2 domains of phospholipase C $\gamma 1$, GAP, and Src to activated growth factor receptors. Science 250: 979-982.

Benson Jr., D.W., Hughes, S.F., Hu, N., and Clark, E.B. 1989. Effect of heart rate increase on dorsal aortic flow before and after volume loading in the stage 24 chick embryo. Pediatr. Res. 26: 438-441.

Berdougo, E., Coleman, H., Lee, D.H., Stainier, D.Y., and Yelon, D. 2003. Mutation of weak atrium/atrial myosin heavy chain disrupts atrial function and influences ventricular morphogenesis in zebrafish. Development 130: 6121-6129.

Braz, J.C., Gregory, K., Pathak, A., Zhao, W., Sahin, B., Klevitsky, R., Kimball, T.F., Lorenz, J.N., Nairn, A.C., Liggett, S.B., et al. 2004. PKC- $\alpha$ regulates cardiac contractility and propensity toward heart failure. Nat. Med. 10: 248-254.

Burggren, W.W. and Pinder, A.W. 1991. Ontogeny of cardiovascular and respiratory physiology in lower vertebrates. Annu. Rev. Physiol. 53: 107-135.

Carmeliet, P., Ferreira, V., Breier, G., Pollefeyt, S., Kieckens, L., Gertsenstein, M., Fahrig, M., Vandenhoeck, A., Harpal, K., Eberhardt, C., et al. 1996. Abnormal blood vessel development and lethality in embryos lacking a single VEGF allele. Nature 380: 435-439.

Chen, J.N. and Fishman, M.C. 1996. Zebrafish tinman homolog demarcates the heart field and initiates myocardial differentiation. Development 122: 3809-3816.

Ferrara, N. 2001. Role of vascular endothelial growth factor in regulation of physiological angiogenesis. Am. I. Physiol. Cell. Physiol. 280: C1358-C1366.

Frank, O. 1959. On the dynamics of cardiac muscle. Am. Heart J. 58: 282-317.

Funamoto, M., Fujio, Y., Kunisada, K., Negoro, S., Tone, E., Osugi, T., Hirota, H., Izumi, M., Yoshizaki, K., Walsh, K., et al. 2000. Signal transducer and activator of transcription 3 is required for glycoprotein 130-mediated induction of vascular endothelial growth factor in cardiac myocytes. J. Biol. Chem. 275: 10561-10566.

Garrity, D.M., Childs, S., and Fishman, M.C. 2002. The heartstrings mutation in zebrafish causes heart/fin Tbx5 deficiency syndrome. Development 129: 4635-4645.

Gerfen, C.R., Choi, W.C., Suh, P.G., and Rhee, S.G. 1988. Phospholipase C I and II brain isozymes: Immunohistochemical localization in neuronal systems in rat brain. Proc. Natl. Acad. Sci. 85: 3208-3212.

Gillebert, T.C., Sys, S.U., and Brutsaert, D.L. 1989. Influence of loading patterns on peak length-tension relation and on relaxation in cardiac muscle. I. Am. Coll. Cardiol. 13: 483490.

Gordon, A.M. and Pollack, G.H. 1980. Effects of calcium on the sarcomere length-tension relation in rat cardiac muscle. Im- plications for the Frank-Starling mechanism. Circ. Res. 47: 610-619.

Gu, C., Rodriguez, E.R., Reimert, D.V., Shu, T., Fritzsch, B., Richards, L.J., Kolodkin, A.L., and Ginty, D.D. 2003. Neuropilin-1 conveys semaphorin and VEGF signaling during neural and cardiovascular development. Dev. Cell 5: 45-57.

Habeck, H., Odenthal, J., Walderich, B., Maischein, H., and Schulte-Merker, S. 2002. Analysis of a zebrafish VEGF receptor mutant reveals specific disruption of angiogenesis. Curr. Biol. 12: 1405-1412.

Hu, N., Sedmera, D., Yost, H.J., and Clark, E.B. 2000. Structure and function of the developing zebrafish heart. Anat. Rec. 260: $148-157$.

Jowett, T. and Lettice, L. 1994. Whole-mount in situ hybridizations on zebrafish embryos using a mixture of digoxigeninand fluorescein-labelled probes. Trends Genet. 10: 73-74.

Kang, M. and Walker, J.W. 2005. Protein kinase C $\delta$ and $\varepsilon$ mediate positive inotropy in adult ventricular myocytes. J. Mol. Cell. Cardiol. 38: 753-764.

Lamers, J.M., De Jonge, H.W., Panagia, V., and Van Heugten, H.A. 1993. Receptor-mediated signalling pathways acting through hydrolysis of membrane phospholipids in cardiomyocytes. Cardioscience 4: 121-131.

Lawson, N.D., Mugford, J.W., Diamond, B.A., and Weinstein, B.M. 2003. phospholipase $C \gamma-1$ is required downstream of vascular endothelial growth factor during arterial development. Genes \& Dev. 17: 1346-1351.

Lee, P., Goishi, K., Davidson, A.J., Mannix, R., Zon, L., and Klagsbrun, M. 2002. Neuropilin-1 is required for vascular development and is a mediator of VEGF-dependent angiogenesis in zebrafish. Proc. Natl. Acad. Sci. 99: 10470-10475.

Li, J., Hampton, T., Morgan, J.P., and Simons, M. 1997. Stretchinduced VEGF expression in the heart. J. Clin. Invest. 100: $18-24$.

Liao, H.J., Kume, T., McKay, C., Xu, M.J., Ihle, J.N., and Carpenter, G. 2002. Absence of erythrogenesis and vasculogenesis in Plcg1-deficient mice. J. Biol. Chem. 277: 9335-9341.

McLaughlin, A.P. and De Vries, G.W. 2001. Role of PLC $\gamma$ and $\mathrm{Ca}^{2+}$ in VEGF- and FGF-induced choroidal endothelial cell proliferation. Am. J. Physiol. Cell. Physiol. 281: C1448C1456.

Michelmore, R.W., Paran, I., and Kesseli, R.V. 1991. Identification of markers linked to disease-resistance genes by bulked segregant analysis: A rapid method to detect markers in specific genomic regions by using segregating populations. Proc. Nat1. Acad. Sci. 88: 9828-9832.

Most, P., Boerries, M., Eicher, C., Schweda, C., Ehlermann, P., Pleger, S.T., Loeffler, E., Koch, W.J., Katus, H.A., Schoenenberger, C.A., et al. 2003. Extracellular S100A1 protein inhibits apoptosis in ventricular cardiomyocytes via activation of the extracellular signal-regulated protein kinase 1/2 (ERK1/ 2). J. Biol. Chem. 278: 48404-48412.

Nasevicius, A., Larson, J., and Ekker, S.C. 2000. Distinct requirements for zebrafish angiogenesis revealed by a VEGF-A morphant. Yeast 17: 294-301.

Neufeld, G., Cohen, T., Gengrinovitch, S., and Poltorak, Z. 1999. Vascular endothelial growth factor (VEGF) and its receptors. Faseb J. 13: 9-22.

Puceat, M. and Vassort, G. 1996. Signalling by protein kinase C isoforms in the heart. Mol. Cell. Biochem. 157: 65-72.

Rottbauer, W., Baker, K., Wo, Z.G., Mohideen, M.A., Cantiello, H.F., and Fishman, M.C. 2001. Growth and function of the embryonic heart depend upon the cardiac-specific L-type calcium channel $\alpha 1$ subunit. Dev. Cell 1: 265-275.

Rottbauer, W., Saurin, A.J., Lickert, H., Shen, X., Burns, C.G., Wo, Z.G., Kemler, R., Kingston, R., Wu, C., and Fishman, M. 
2002. Reptin and pontin antagonistically regulate heart growth in zebrafish embryos. Cell 111: 661-672.

Schaldach, M. and Hutten, H. 1990. A physiological approach to different concepts of rate adaptive cardiac pacing. Med. Prog. Technol. 16: 235-246.

Seko, Y., Takahashi, N., Tobe, K., Ueki, K., Kadowaki, T., and Yazaki, Y. 1998. Vascular endothelial growth factor (VEGF) activates Raf-1, mitogen-activated protein (MAP) kinases, and S6 kinase (p90rsk) in cultured rat cardiac myocytes. J. Cell. Physiol. 175: 239-246.

Seko, Y., Takahashi, N., Shibuya, M., and Yazaki, Y. 1999. Pulsatile stretch stimulates vascular endothelial growth factor (VEGF) secretion by cultured rat cardiac myocytes. Biochem. Biophys. Res. Commun. 254: 462-465.

Shalaby, F., Rossant, J., Yamaguchi, T.P., Gertsenstein, M., Wu, X.F., Breitman, M.L., and Schuh, A.C. 1995. Failure of bloodisland formation and vasculogenesis in Flk-1-deficient mice. Nature 376: 62-66.

Shibuya, M., Seetharam, L., Ishii, Y., Sawano, A., Gotoh, N., Matsushime, H., and Yamaguchi, S. 1994. Possible involvement of VEGF-FLT tyrosine kinase receptor system in normal and tumor angiogenesis. Princess Takamatsu Symp. 24: $162-170$.

Sonnenblick, E.H., Skelton, C.L., Spotnitz, W.D., and Feldman, D. 1973. Redefinition of the ultrastructural basis of cardiac length-tension relations. Circ 48: 65.

Stainier, D.Y. and Fishman, M.C. 1994. The zebrafish as an model system to study cardiovascular development. Trends Cardiovasc. Med. 4: 207-212.

Stainier, D.Y., Fouquet, B., Chen, J.N., Warren, K.S., Weinstein, B.M., Meiler, S.E., Mohideen, M.A., Neuhauss, S.C., SolnicaKrezel, L., Schier, A.F., et al. 1996. Mutations affecting the formation and function of the cardiovascular system in the zebrafish embryo. Development 123: 285-292.

Starling, E.H. 1918. Linacre lecture on the law of the heart. Longmans, Green and Co., London, UK.

Takahashi, T., Yamaguchi, S., Chida, K., and Shibuya, M. 2001. A single autophosphorylation site on KDR/Flk-1 is essential for VEGF-A-dependent activation of PLC- $\gamma$ and DNA synthesis in vascular endothelial cells. EMBO J. 20: 2768-2778.

Thompson, M.A., Ransom, D.G., Pratt, S.J., MacLennan, H., Kieran, M.W., Detrich III, H.W., Vail, B., Huber, T.L., Paw, B., Brownlie, A.J., et al. 1998. The cloche and spadetail genes differentially affect hematopoiesis and vasculogenesis. Dev. Biol. 197: 248-269.

Van Henningen, R., Rijinsburger, W.H., and ter Keurs, H.E.D.J. 1982. Sarcomere length control in striated muscle. Am. J. Physiol. 242: H411.

Westerfield, M. 1995. The zebrafish book: A guide for the laboratory use of zebrafish Danio rerio. Eugene, OR.

Wood, J.M., Bold, G., Buchdunger, E., Cozens, R., Ferrari, S., Frei, J., Hofmann, F., Mestan, J., Mett, H., O'Reilly, T., et al. 2000. PTK787/ZK 222584, a novel and potent inhibitor of vascular endothelial growth factor receptor tyrosine kinases, impairs vascular endothelial growth factor-induced responses and tumor growth after oral administration. Cancer Res. 60: 2178-2189.

Yamaguchi, S., Iwata, K., and Shibuya, M. 2002. Soluble Flt-1 (soluble VEGFR-1), a potent natural antiangiogenic molecule in mammals, is phylogenetically conserved in avians. Biochem. Biophys. Res. Commun. 291: 554-559.

Yelon, D., Horne, S.A., and Stainier, D.Y. 1999. Restricted expression of cardiac myosin genes reveals regulated aspects of heart tube assembly in zebrafish. Dev. Biol. 214: 23-37.

Yule, D.I. and Williams, J.A. 1992. U73122 inhibits Ca2+ oscil- lations in response to cholecystokinin and carbachol but not to JMV-180 in rat pancreatic acinar cells. J. Biol. Chem. 267: 13830-13835. 


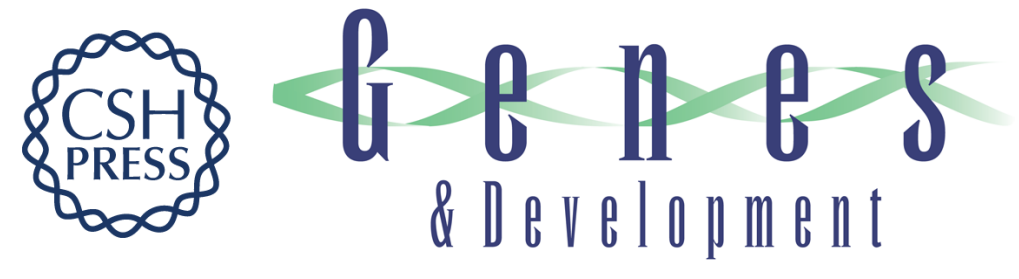

\section{VEGF-PLC $\gamma 1$ pathway controls cardiac contractility in the embryonic heart}

Wolfgang Rottbauer, Steffen Just, Georgia Wessels, et al.

Genes Dev. 2005, 19:

Access the most recent version at doi:10.1101/gad.1319405

Supplemental
Material http://genesdev.cshlp.org/content/suppl/2005/06/16/19.13.1624.DC1

References This article cites 45 articles, 17 of which can be accessed free at: http://genesdev.cshlp.org/content/19/13/1624.full.html\#ref-list-1

\section{License}

Email Alerting

Service

Receive free email alerts when new articles cite this article - sign up in the box at the top right corner of the article or click here.

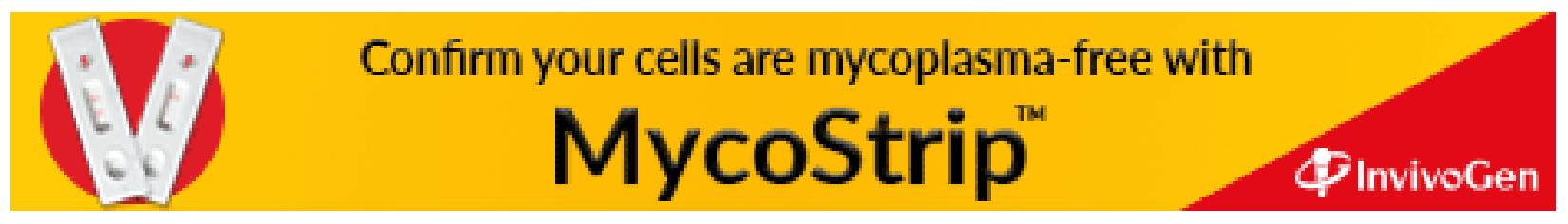

